\title{
Information choices for navigation
}

\section{Breed ${ }^{1}$}

Published online: 11 October 2018

(c) International Union for the Study of Social Insects (IUSSI) 2018
What happens when an animal has choices of navigational information from two or more sources? In foraging social insects, this choice often occurs when workers have the decision to use either learned navigational cues and pheromone trails or markers. Using learned cues may involve trade-offs between speed and accuracy, as movements oriented to landmarks are usually faster than movements based on continuous search for pheromone cues, but the workers may be less prone to errors when following pheromone trail.

An added wrinkle to this trade-off is that the accuracy of landmark-based navigation usually improves with experience. An animal that has navigated a route several times may move more confidently, accurately, and rapidly than a naïve individual. This experiential shift can also represent a gradual shift from the use of social cues, such as pheromones, to internalized landmark memory (which can be considered private information), with some reliance on both types of cues during intermediate stages.

In this issue, Middleton, Reid, and their colleagues investigate these shifts in information usage in Australian meat ants, Iridomyrmex purpureus, using a y-maze experimental design (Middleton et al. 2018). They found that, as with other species of ants, experienced workers use landmark information in preference to trail pheromone information, and that if the trail information is removed, experienced workers continue to be able to navigate the route. The memorized route information is considered private (internal to the individual) while the pheromone information is public (available to all colony members).

A unique element of this study is the discovery that the meat ant workers' performance using private information does not improve with repeated experience; they do well with private information after just navigating the route once. Regarding learning style, this rapid acquisition of

M. Breed

michael.breed@colorado.edu

1 Department of Ecology and Evolutionary Biology, The University of Colorado, Boulder, Boulder, CO 80309-0334, USA information matches well with imprinting, in which an animal rapidly acquires critical information. This pattern differs from the learning curve, showing improvement over trials, that typically results from trial and error learning. This finding supports the critical role of navigational accuracy in successful social insect worker foraging. It would be fascinating in future studies to further explore the speed with which navigational route is acquired by ants and to compare those results with the much more thoroughly studied honey bee.

Because foraging by social insects is usually a cooperative, rather than a competitive, venture for workers from the same colony, the concept of private versus public information applies differently than in other behavioral contexts. In systems involving competition, including attraction of mates or food search by non-cooperating animals, information is held privately because it has particular value to the holder, and that value would be compromised if other animals, by eavesdropping or spying, capture the information.

The use of the public/private categorization in this context, though, is intriguing because it raises the question of at what point would a forager become motivated to share its private information publicly, by re-laying pheromone trail or communicating an alternative more direct route to the goal? This question leads to additional possible future directions, with studies focused on the mechanisms of choice between continuing to forage and shifting to providing social information to colony-mates.

Michael Breed, Editor-in-Chief.

\section{Reference}

Middleton EJT, Reid CR, Mann RP, Latty T (2018) Social and private information influence the decision making of Australian meat ants (Iridomyrmex purpureus). Insect Soc. https://doi.org/10.1007/ s00040-018-0656-1 